\title{
La Colección Nacional de Aves del Museo de Historia Natural de El Salvador: 40 años de ciencia
}

Ricardo Ibarra Portillo

\section{Antecedentes}

Durante mi estadía laboral en el Ministerio de Medio Ambiente y Recursos Naturales (MARN) se realizó, bajo convenio con el Museo de Historia Natural de El Salvador (Muhnes), la revisión y ordenamiento de la Colección Nacional de Aves (CNA), que incluyó la digitalización de la información que acompaña a cada uno de los ejemplares que conforman dicha colección, elaborándose así una base de datos en Excel. Es a partir de estos datos que se elaboró el presente artículo.

\section{Introducción}

Al entrar y recorrer la exhibición de aves del Muhnes, muy probablemente los visitantes, mientras contemplen las diferentes especies montadas en los dioramas y con sus rótulos informativos, no dimensionen qué hay mas allá o del porqué de estos diseños, cuánta gente está involucrada en que tal ave esté en un bosque o que otra haya sido puesta en el suelo o caminando a la orilla del agua. Todo este trabajo es producto del conocimiento que se tiene de las aves mismas, ya sea mediante libros de consulta como guías de campo y volúmenes más especializados o de las aves como tales. Asimismo, de los datos que aportan al ser colectadas, como el estado de su plumaje, el contenido estomacal y los parásitos que poseía, entre otros más.

En este sentido, la recolecta o colecta científica es importante, aunque también es un proceder comúnmente mal visto $\mathrm{y}$, por ende, mal interpretado por el público en general e incluso por algunos profesionales en la materia (Stiles, 2002), ya que se juzga el acto de quitarle la vida a un ser solo para guardarlo 
en un museo sin utilidad evidente. Sin embargo, las colecciones de fauna, flora, minerales, fósiles y muchas cosas más, constituyen un verdadero tesoro para todo país ávido de conocer y conservar su patrimonio natural, pues, bajo una efectiva administración, las colecciones científicas son la columna vertebral en diversas aplicaciones al momento de tomar decisiones y llevar a cabo proyectos, líneas base, planes o programas.

El presente artículo tiene como objetivo contribuir en dar a conocer, a todo interesado en la ciencia y conservación en El Salvador, la información contenida en la CNA del Muhnes. Es además el fruto de la coordinación, que entre 2004 y 2007, se realizó entre la Dirección General de Patrimonio Natural del MARN y la Dirección del Muhnes para tener acceso a dicho acervo científico para digitalizar los datos de los especímenes.

\section{Quiénes, cómo y por qué hacer una recolecta científica}

Hay que considerar que la recolecta científica requiere de una alta dosis de responsabilidad y debe, además, estar enfocada hacia un fin determinado desde el que se contribuya a la ciencia, aparte de la formación profesional que está implícita (Stiles, 2002). Aunque también un particular puede realizar recolecta científica, si se le presenta la oportunidad con un animal, para el caso, un ave muerta que encuentre cerca de su vecindario, lugar de trabajo o en algún recorrido que realice e irla a donar el Muhnes con la información sobre su procedencia.

\section{Importancia de las colecciones biológicas}

Las colecciones biológicas se forman de numerosas recolectas científicas, que se traducen en especímenes organizados con un orden establecido y continuos cuidados. De acuerdo con Waddington, 1989; Dankz, 1991 y Romo, 1991, citados por Ramírez-Albores et al. 2002, estando adecuadamente preservados permiten realizar, entre otras cosas, clasificación taxonómica, determinación de la distribución de los organismos, descripción de especímenes, elaboración de listados faunísticos, claves e ilustraciones para su identificación, determinación y asociación de especies, análisis biogeográficos y ecológicos y almacenamiento en bases de datos computarizadas. 


\section{Marco legal}

$\mathrm{Al}$ respecto, es necesario conocer el marco legal vigente, que es la Ley de Conservación de Vida Silvestre, que en su Capítulo III, Art. 8, menciona que la recolecta de vida silvestre estará normada por los reglamentos correspondientes y administrados por el MARN, en coordinación con los organismos o instituciones relacionados con la materia; y el Art. 26 reza que constituye una infracción grave el capturar ejemplares de vida silvestre con fines científicos u otros sin el permiso correspondiente (MARN, 2001).

Asimismo, las colecciones ya conformadas están normadas por la Ley del Patrimonio Cultural, que reza, en el Artículo 3, que una vez ingresado un espécimen a la CNA del Muhnes, este está bajo el cuido y conservación de la Secretaría de Cultura de la Presidencia y forma parte de los bienes culturales de El Salvador.

\section{Las colecciones ornitológicas en la región centroamericana y Panamá}

En Centroamérica y Panamá, de acuerdo con Escalante Pliego (2005), ${ }^{1}$ la información de pieles de aves en museos u otras instituciones consiste de las siguientes colecciones: Costa Rica, 13.301 pieles (entre el Museo de Historia Natural de Costa Rica y la Colección de Aves del Museo de Zoología de la Universidad de Costa Rica); Guatemala, 620 (de la Universidad del Valle); Panamá, 4.506 (entre la Colección Conmemorativa Gorgas, el Museo de Vertebrados de Panamá y el Museo de Ciencias Naturales); y en El Salvador, 811 (del Munhes). ${ }^{2}$

\section{Breve historia del estudio de aves en EI Salvador}

En El Salvador, a pesar de que se han realizado varias iniciativas para documentar la avifauna desde finales del siglo XVIII y principios del XIX (colectas en el golfo de Fonseca por buques extranjeros; Dickey\& van Rossem [1938], Marshall [1943] y Steinbacher[1958], lastimosamente no se cuenta con especímenes de referencia producto de esos estudios en el país. Estos se encuentran, en su mayoría, depositados en el Museo de Historia Natural de Chicago, Illinois, Estados Unidos, y el Museo del Instituto Seckenberg en Berlín, Alemania.

1 http://www.aou.org/committees/collections/docs/Colecciones\%20Ornitol\%F3gicas\%201a\%20 Regi\%F3n\%20Neotropical\%20205.doc.

2 Este dato no concuerda con lo expuesto en el presente documento. 
En la década de 1970 se creó el Museo de Historia Natural de El Salvador (Muhnes), al igual que el entonces Servicio de Parques Nacionales y Vida Silvestre (Panavis), y fue así como se llevó a cabo trabajo intenso en campo en diferentes partes del país por profesionales nacionales, principalmente en áreas naturales como el Parque Nacional de Montecristo en Santa Ana; el cerro Cacahuatique en Morazán; la laguna El Jocotal en San Miguel; el golfo de Fonseca en La Unión y el Parque Nacional El Imposible en Ahuachapán.

Otros esfuerzos enriquecieron aún más la colección de ornitología de El Salvador, como Thurber et al. (1987), Figueroa de Tobar (1993), el Inventario y Taxonomía de las Aves de El Salvador (Komar, Com. Pers.) entre 2001-2004, y últimamente el Proyecto de Monitoreo de Aves de El Salvador desarrollado por SalvaNATURA desde 2003 a la fecha.

\section{La colección ornitológica de El Salvador}

Al mes de junio de 2007, la colección contaba con 786 pieles de especies que ocurren en El Salvador y catorce pieles de diez especies exóticas producto del intercambio con otros museos como el de Ontario, en Canadá, y otras pieles traídas de Georgia y Florida, Estados Unidos (30); Guatemala y Nicaragua, totalizando 800 pieles.

La gran mayoría de pieles provienen de colectas realizadas durante la década de 1970 (75\%), 1980 (11\%), 2000 (6\%) y 1990 (4\%); un 5\% más no presenta información de fechas. La piel más antigua es de un "pavito" (Myioborusminiatus) colectado el 15 de abril de 1970 en el Parque Nacional Montecristo, departamento de Santa Ana. En general, las pieles proceden de 23 sitios de todo el país, pero son diez los que representan más de la mitad del total (66\%) de las pieles de la colección. Esto refleja el trabajo realizado por los técnicos del entonces Panavis.

Tabla 1. Pieles y porcentajes del total para los diez sitios principales

\begin{tabular}{lcc}
\hline Sitio & No. de pieles & $\begin{array}{l}\text { Porcentaje del } \\
\text { total }\end{array}$ \\
\hline Laguna El Jocotal & 92 & 18 \\
P. SaburoHirao & 87 & 17 \\
P. N. El Imposible & 85 & 16 \\
P. N. Montecristo & 80 & 15 \\
C. Cacahuatique & 62 & 12 \\
ZoológicoNacional & 38 & 7 \\
Barra de Santiago & 27 & 5 \\
P. N. Los Volcanes & 20 & 4 \\
L. de Coatepeque & 15 & 3 \\
Golfo de Fonseca & 11 & 2 \\
\hline Total & $\mathbf{5 1 7}$ & $\mathbf{9 6}$ \\
\hline
\end{tabular}


Las 786 pieles pertenecen a 267 especies, que representan el $48 \%$ del total de especies registradas para El Salvador (555, Ibarra Portillo, Com. Pers. diciembre 2009), e incluyen 53 familias de 18 órdenes. Las familias más representadas son: Parulidae con 22 especies y 103 pieles (13\%), Turdidae con 12 especies y 59 pieles (8\%), Tyrannidae con 25 especies y 56 pieles (7\%), Trochilidae con 15 especies y 50 pieles (6\%) y Anatidae con 6 especies y 41 pieles (5\%).

Las diez especies con mayor cantidad de pieles son: "pato enmascarado" (Nomonyxdominicus) (19), "chonte" (Turdusgrayi) (16), "chipe" (Basileuterusrufifrons) (13), "gallineta pico blanco" (Fúlica americana) (10), "colibrí morado" (Campylopterushemileucurus) (10), "zorzal de Swainson" (Catharusustulatus) (11), "puñalada" (Pheucticusludovicianus) (11), "tordito" (Molothrusaeneus) (11) y "chiltota espalda rayada" (Icteruspustulatus) (10), que representan en conjunto el $15 \%$ del total de pieles del Muhnes.

\section{Especies interesantes}

Entre las especies más interesantes y de alto valor científico, ya sea porque son especies raras, fueron nuevos registros en su momento para el país o son de las que se cuenta con pocos registros a lo largo del tiempo, se mencionan: una serie de 19 individuos de "pato enmascarado" (Nomonyxdominicus) colectados entre marzo y julio de 1977 en la laguna El Jocotal, San Miguel; un "rabijunco" (Phaethonaethereus) de Barra de Santiago; un "zope cabeza amarilla" ( $\mathrm{Ca}$ thartesburrovianus) delcerro Cacahuatique; dos individuos de "rálido blanco y negro" (Pardirallusmaculatus) (isla Tasajera, estero de Jaltepeque y laguna El Jocotal); dos individuos de "golondrina de mar" (Onychoprionanaethetus) de Los Farallones, golfo de Fonseca; dos individuos de "rayador americano" (Rynchopsniger) de la laguna El Jocotal; una "paloma de bosque" (Geotrygon montana) del Parque Nacional El Imposible; un "lechuza de la madriguera" (Athenecanicularia) de una finca en La Libertad; un "búho fulvo" (Strixfulvescens) de Parque Nacional de Montecristo; un "pavita" (Hylomanesmomotula) del Parque Nacional El Imposible; un "carpintero enano" (Veniliornisfumigatus) de la Costa del Sol; un "mosquero de collar" (Xenotriccuscallizonus) de Parque Nacional Montecristo y un individuo de los "chipes" (Dendroicatigrina y $D$. castanea), pero sin procedencia.

Últimamente, y como producto de corrientes marinas desde Suramérica, un individuo de "pingüino de Magallanes" (Spheniscusmagellanicus) fue arrastrado desde la parte sur del continente y apareció en la bocana El Saite, sector este 
de Barra de Santiago, Ahuachapán, el 7 de junio de 2007, y murió posteriormente por debilidad. La piel fue depositada en el Muhnes y constituye el primer registro de este tipo de aves en El Salvador.

\section{Proporción por departamento}

Los cuatro departamentos con mayor porcentaje de pieles son: San Salvador con 208 (27\%), Ahuachapán con 139 (18\%), Santa Ana con 118 (15\%) y San Miguel con 109 (14\%). Cabe aclarar que, en el caso de San Salvador, la mayoría (60\%) proviene del parque SaburoHirao y del parque Zoológico Nacional. En total de todas las pieles de los departamentos registrados alcanza 96\%, el $4 \%$ restante (30 pieles) no presenta datos de localidad (tabla 2).

Tabla 2. Total de pieles por departamento y porcentaje del total

\begin{tabular}{lcc}
\hline Departamento & No. de pieles & $\begin{array}{l}\text { Porcentaje del } \\
\text { total }\end{array}$ \\
\hline San Salvador & 208 & 28 \\
Ahuachapán & 139 & 18 \\
Santa Ana & 118 & 16 \\
San Miguel & 109 & 14 \\
Morazán & 77 & 10 \\
La Libertad & 30 & 4 \\
Chalatenango & 18 & 2 \\
Sonsonate & 18 & 2 \\
La Paz & 17 & 2 \\
La Unión & 11 & 1 \\
San Vicente & 5 & 0,6 \\
Usulután & 3 & 0,4 \\
Cabañas & 1 & 0,1 \\
Cuscatlán & 1 & 0,1 \\
\hline Total & $\mathbf{7 5 6}$ & $\mathbf{9 6}$ \\
\hline
\end{tabular}

Del total de pieles depositadas en el Muhnes, solamente 115 (15\%) tienen datos completos, esto en lo referente al código de ingreso, nombre científico de la especie, sexo, edad, fecha, localidad, medidas, colector y de datos de campo complementarios.

\section{Conclusiones}

La CNA del Muhnes tiene un incalculable valor científico y representa igualmente un bien cultural que pertenece a todos los salvadoreños. Cumplió 40 años en 2010, y constituye una fiel muestra del trabajo realizado por una amplia gama de profesionales que se han preocupado, a lo largo de todo este 
tiempo, por documentar este grupo de fauna en El Salvador, y se ve reflejado en la amplia variedad de especies (277) y pieles de estudio (800), así como información sobre especies raras que allí se mantiene, quedando planteado dicho desafío para los años venideros.

El apoyo del Estado es importante para la conservación de la CNA del Muhnes, en el sentido de asegurar la perdurabilidad de las pieles de estudio $\mathrm{y}$, de igual modo, el promulgar el permanente mejoramiento en el manejo, las condiciones de su preservación, mantenimiento y continuo enriquecimiento.

\section{Agradecimientos}

A las licenciadas Eunice Echeverría y Ana María Quevedo, así como también a Hugo Villalta por su colaboración a lo largo del proceso coordinado entre el MARN y el Muhnes, y al Dr. Ramón Rivas, de la Secretaría de Cultura, por su respaldo institucional para la presente investigación.

\section{Referentes bibliográficos}

MARN. Ley de Conservación de Vida Silvestre. Julio. Decreto No. 844. 2001.

Dickey D. \& A.J. van Rossem. The Birds of El Salvador, Zoological Series, Field Museum of Natural History. Chicago (23) pp. 406-609. 1938.

Figueroa de Tobar, M.C. Aves acuáticas costeras de Barra de Santiago, Ahuachapán. Publicaciones Ocasionales No. 3, Museo de Historia Natural de El Salvador. Noviembre. 1993.

Marshall, Jr. J.T. Aditional Information concerning the Birds of El Salvador, Condor 45:21-33. 1943. 
Ramírez-Albores, J.E.; G. Ramírez y A. Bueno. "Huitzil, la colección ornitológica del Museo de Zoología de la Facultad de Estudios Superiores”, Zaragoza, Unam. Vol. 3 (1): 28-32. 2002.

Steinbacher, J. WeitereBeitragezurVogelfauna von El Salvador. Senck. Biol. , 39: 11-40. 1958.

Stiles, G. BioByte, Project BioMap, September, 3:1-2. 2002.

Thurber, W.A.; Serrano J.F.; Sermeño, A \& Benítez, M. Status of uncommon and previously Unreported Birds of El Salvador. Proceeding of the Western Foundation of the Vertebrate Zoology, Los Angeles, California, Vol. 3 (3): 109-293. 1987. 


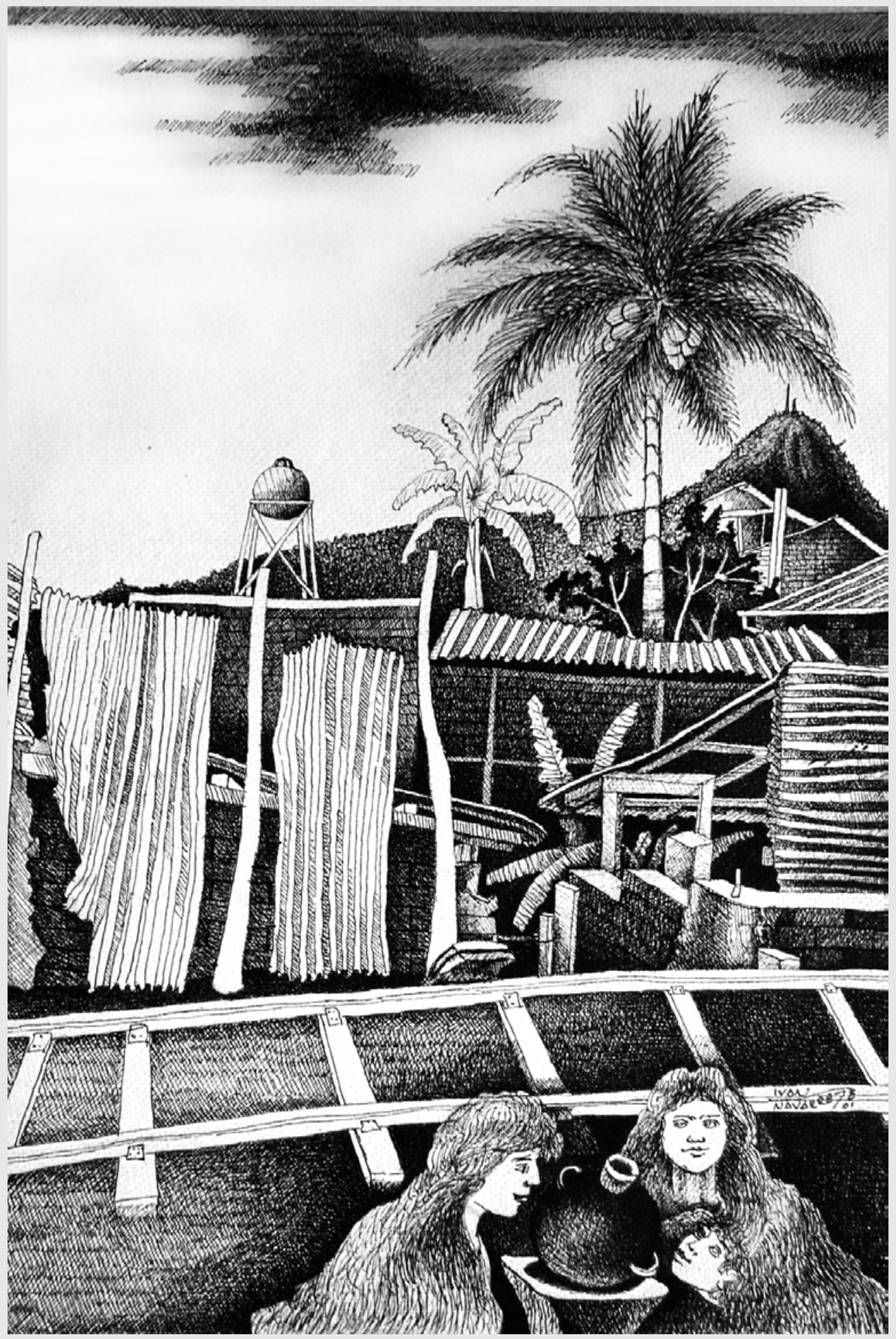

